\title{
The Cre1 and Cre3 Nematode Resistance Genes Are Located at Homeologous Loci in the Wheat Genome
}

\author{
John de Majnik,, ${ }^{1,4}$ Francis C. Ogbonnaya, ${ }^{2,3}$ Odile Moullet, ${ }^{1}$ and Evans S. Lagudah ${ }^{1}$ \\ ${ }^{1}$ CSIRO Plant Industry, GPO Box 1600, Canberra, ACT 2601, Australia; ${ }^{2}$ Department of Primary Industries, Victorian \\ Institute for Dryland Agriculture, Natimuk Road, PB 260, Horsham, VIC $3401,{ }^{3}$ The University of Melbourne, Joint Centre \\ for Crop Innovation, Parkville, VIC 3052, Australia; ${ }^{4}$ Graingene, 65 Canberra Avenue, Griffith ACT 2603, Australia
}

Submitted 10 July 2003. Accepted 4 August 2003.

\begin{abstract}
Differential responses in host-nematode pathotype interactions occur in wheat lines carrying different cereal cyst nematode resistance $(\mathrm{Cre})$ genes. $\mathrm{Cre1}$, located on chromosome $2 \mathrm{~B}$, confers resistance to most European nematodes and the sole Australian pathotype, while $\mathrm{Cre3}$, present on chromosome 2D, is highly resistant to the Australian pathotype and susceptible to a number of European pathotypes. Genes encoding nucleotide binding site-leucine rich repeat (NBS-LRR) proteins that cosegregate with the Cre3 locus cross hybridize to homologues whose restriction fragment length polymorphism (RFLP) patterns distinguish near-isogenic $\mathrm{Cre} 1$ nematode-resistant wheat lines. Genetic mapping showed that the NBS-LRR gene members that distinguished the Cre1 near-isogenic lines were located on chromosome 2BL at a locus, designated $X c s l 107$, that cosegregates with the Cre1 locus. A haplotype of NBS-LRR genes from the Xcsl107 locus provides a diagnostic marker for the presence of $\mathrm{Cre} 1$ nematode resistance in a wide collection of wheat lines and segregating families. Genetic analysis of NBS-LRR haplotypes that cosegregate with $\mathrm{Crel}$ and $\mathrm{Cre3}$ resistance, together with flanking cDNA markers and other markers from homoeologous group 2 chromosomes, revealed a conserved gene order that suggests $\mathrm{Cre} 1$ and $\mathrm{Cre} 3$ are homeoloci.
\end{abstract}

Resistance to cereal cyst nematode (Heterodera avenae) was first reported in barley (Nilsson-Ehle 1920), and four decades later, was also described in bread wheat (Nielsen 1966). The resistance trait from bread wheat was designated $\mathrm{Cre}$. Additional sources of resistance to cereal cyst nematode $(\mathrm{CCN})$ have been identified among the wild relatives of wheat (Ogbonnaya et al. 2001b). Of the nine resistance genes ( $\mathrm{Crel}, 2,3,4,5,6,7, \mathrm{~F}$, and $R$ ) described so far in the Triticeae, based on pathotype specificity and independent chromosomal location, only two (Crel and $\mathrm{CreF}$ ) are derived directly from bread wheat (Triticum aestivum). The founder lines for Crel are the bread wheat cultivar Loros and the landrace AUS10894 (O'Brien et al. 1980; Slootmaker et al. 1974). Crel confers resistance to several European $H$. avenae pathotypes as well as the Australian pathotype, albeit with varying levels of nematode reproduction in different genetic backgrounds of the host. Comparison of Crel with the nematode resistance gene $\mathrm{Cre} 3$, derived from the diploid D genome progenitor of wheat, Aegilops tauschii, shows that both provide resistance to the Australian pathotype, but differ in their specific-

Corresponding author: E. Lagudah; E-mail: evans.lagudah@csiro.au

Current address of O. Moullet: Station Federale de Recherche en Production Vegetale de Changins H-1260 Nyon, Switzerland ity to European and Middle Eastern pathotypes (Rivoal et al. 2001). Crel and Cre3 are located on the long arms of chromosomes 2B and 2D, respectively (Eastwood et al. 1994; Williams et al. 1994).

Two distinct classes of genes have been demonstrated to confer nematode resistance in plants: the $\mathrm{Hs}_{s} \mathrm{pro-l}^{\text {gene from a }}$ wild relative of sugar beet (Cai et al. 1997) and genes encoding proteins with nucleotide binding site-leucine rich repeat (NBSLRR) motifs, Mi, Gpa2, and Hero, present on introgressed segments from wild relatives of tomato and potato, which confer resistance against root knot and cyst nematodes (Ernst et al. 2002; Milligan et al. 1998; Van der Vossen et al. 2000). A small family of NBS-LRR sequences ("G4" being the founder sequence) cosegregating with the Cre3 locus on chromosome 2DL have previously been documented in wheat (Lagudah et al. 1997). Closely related homologs of the G4 sequence were also found at syntenic locations on $2 \mathrm{BL}$ as well as nonhomeologous positions in the wheat genome (Spielmeyer et al. 1998). Some of the homologs that mapped outside the Cre3 locus occurred in regions of the genome that are known to carry other cyst nematode resistance loci. For example, G4 related sequences were found in introgressed segments carrying Cre 5 (formerly $\mathrm{CreX}$ ) and $\mathrm{Cre} 6$ resistance genes (Seah et al. 2000; Ogbonnaya et al. 2001a).

Monosomic analysis of the wheat genome was first used to locate Crel nematode resistance to chromosome 2B (Slootmaker et al. 1974). Williams and associates (1994) placed Crel in an interval on chromosome 2BL; however, this placement lacked precision, since it was derived from a small mapping family. Thus, the precise location of Crel remains to be ascertained. We have previously shown, from a limited set of wheat lines, that NBS-LRR gene sequences from the G4 family detected homologues that were diagnostic for Crel nematode resistance (Lagudah et al. 1998). At least three independent loci containing homologs closely related to the G4 sequence were reported on chromosome 2BL by Spielmeyer and associates (1998) in a wheat mapping family that lacked Crel nematode resistance; hence, it was not possible to assign any of the NBS-LRR loci specifically to Cre1. In this study, G4-related NBS-LRR gene members that distinguish Crel near-isogenic lines were isolated and mapped in a segregating family to a locus designated Xcsl107, which cosegregates with Crel. A combination of DNA markers on chromosome 2BL and NBS-LRR gene members cosegregating with the Crel locus were used to establish the precise location of Crel nematode resistance. Conservation of cosegregating genes between $\mathrm{Cre} 1$ and $\mathrm{Cre} 3$, as well as map locations of tightly linked restriction fragment length polymorphism (RFLP) markers, suggest both nematode resistance 
genes are located at homeologous positions on chromosomes 2BL and 2DL, respectively.

\section{RESULTS}

Cre1 "near-isogenic lines" can be distinguished by the G4 sequence family.

Backcrossing the Crel-resistant trait from the donor lines AUS10894 (parent 1) and Loros (parent 2) into the susceptible wheat cultivar Prins (recurrent parent) seven times produced three separate near-isogenic lines (NIL), Prins/10894, Prins/Loros, and the recurrent parent. RFLP analysis on the NIL was conducted using probes from the G4 sequence family. We previously isolated NBS-LRR gene sequences designated G4 and G12 cosegregating with the Cre3 locus (Lagudah et al. 1997; Moullet et al. 1999). In this study, a related gene, G035 (85\% nucleic acid identity to G4), and its corresponding fulllength cDNA (GenBank accession numbers AAC05834 and AY124651) was isolated from the diploid D genome Aegilops tauschii, which enabled the demarcation of probes according to the exon region (Fig 1). Probes from the $5^{\prime}$ and $3^{\prime}$ regions corresponding to each of the two exons (Fig. 1) from G035-5' and a partial cDNA of G035 containing all of exon 2 and G035-3' were used to detect diagnostic RFLP between the Cre1 NIL

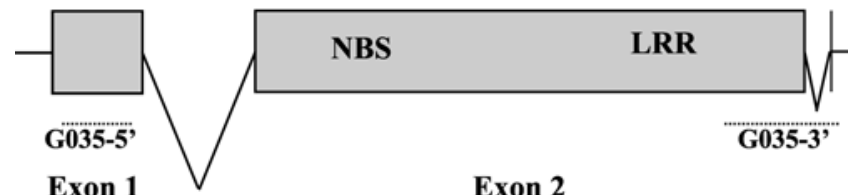

Fig. 1. Diagram of the G035 gene member (GenBank accession number AY124651) related to the G4 sequence. The gene from the predicted translation start to the end is $3,563 \mathrm{bp}$ in length and contains two introns. The size of the first intron is $705 \mathrm{bp}$ and the second one is $92 \mathrm{bp}$. G035 encodes a deduced protein of 921 amino acid residues (GenBank accession number AAM94164). Exon 1 contains a predicted coiled coil domain. The dashed lines indicate regions of exons 1 and 2 used as probes and are referred to as G035-5' and G035-3', respectively. Probe sizes are 488 and 834 bp for G035-5' and G035-3', respectively. The probe from a partial cDNA of G035 spans the entire exon 2 to the $3^{\prime}$ end of the gene. The filled boxes indicate coding regions, and angled lines indicate intron positions. and parents (Fig. 2). Probes from the corresponding regions of exons 1 and 2 found in G4 and G12 used as probes gave identical RFLP (data not shown). Probes from G035-5' and the partial cDNA of G035 identified a maximum of four different polymorphic fragments in DraI-, NdeI-, and XbaI-digested genomic DNA, which revealed diagnostic fragments associated with Crel (Table 1). In addition, fragments were identified in the recurrent susceptible parent that were absent in the resistant line and that probably correspond to the allelic variants from the susceptible parent (Fig. 2).

\section{Cre1 nematode-resistant wheat cultivars carry a unique haplotype.}

The diagnostic fragments in the NIL were further examined in a wider collection of wheat cultivars. A survey of known Crel-resistant and -susceptible wheat varieties from Australia and Europe were analyzed using genomic DNA hybridization to determine the significance of correlation of the polymorphic bands (Fig. 2) to the Crel phenotype. In the six restriction

Table 1. Probe and restriction endonuclease combinations for diagnostic restriction fragment length polymorphisms associated with $\mathrm{Cre}$ 1-resistant plants

\begin{tabular}{|c|c|c|c|c|}
\hline \multirow[b]{2}{*}{ Endonuclease } & \multicolumn{2}{|c|}{ G035-5' } & \multicolumn{2}{|c|}{$\operatorname{Exon}^{x}$} \\
\hline & Size in $\mathbf{k b}^{\mathbf{y}}$ & $\lambda$ clone $^{b}$ & Size in kb & $\lambda$ clone $^{z}$ \\
\hline \multirow[t]{4}{*}{ DraI } & 9.0 & & 15.0 & M59 \\
\hline & 4.5 & S33 & 3.7 & M19 \\
\hline & 2.7 & M19 & 2.0 & \\
\hline & $(0.65)$ & & & \\
\hline \multirow[t]{4}{*}{ NdeI } & 9.5 & S33 & 5.0 & M59 \\
\hline & 4.8 & & 3.8 & S33 \\
\hline & 2.0 & & & \\
\hline & $(0.6)$ & & & \\
\hline \multirow[t]{3}{*}{ XbaI } & 6.1 & S33 & 20.0 & M59 \\
\hline & 3.5 & & 6.5 & \\
\hline & 1.1 & S33 & 2.3 & S33 \\
\hline
\end{tabular}

${ }^{\mathrm{x}}$ The probe from the partial cDNA of G035 that contains all of exon2 and G035-3'.

y Bands in parenthesis can be seen on longer gels.

${ }^{\mathrm{z}}$ M19, M59, and S33 are designated names for genomic lambda clones containing the corresponding diagnostic fragment sizes associated with Cre1-resistant plants.
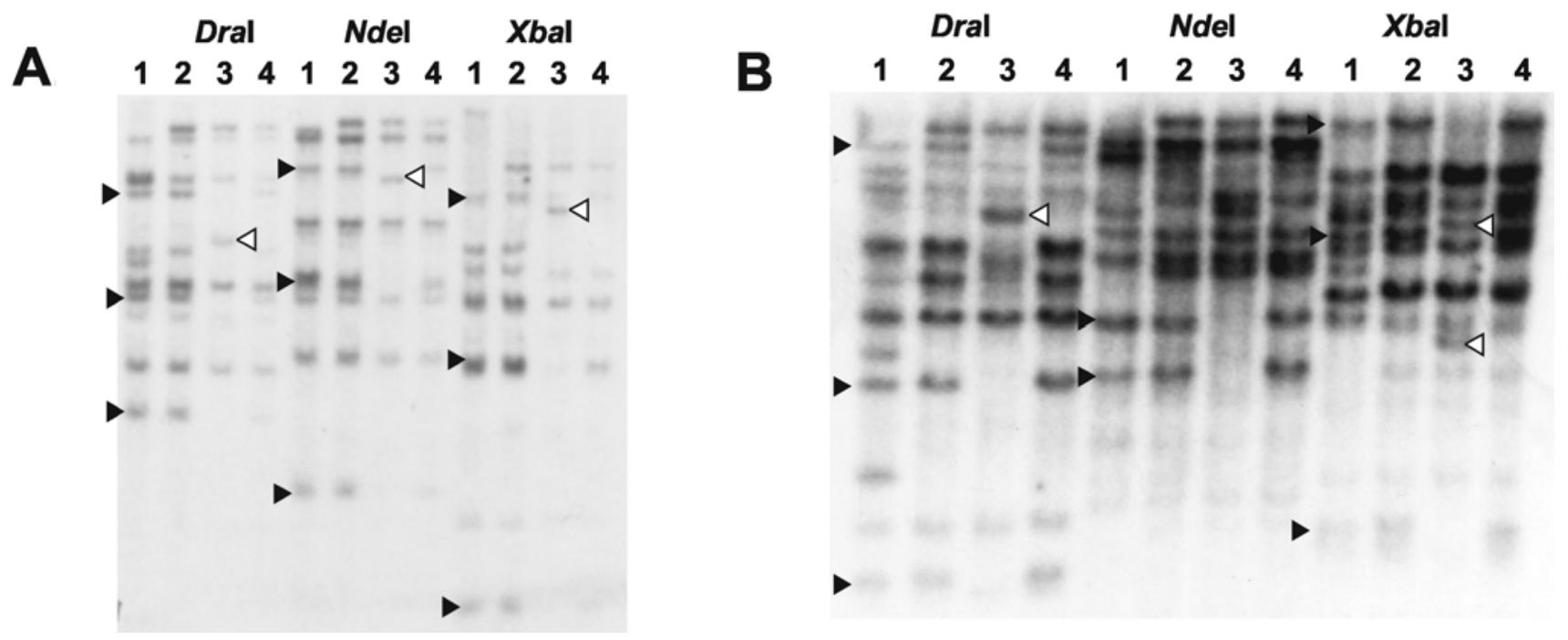

Fig. 2. Hybridization patterns of genomic DNA blots from near-isogenic lines (NIL) and their parents with sequences from G035. Lane 1, AUS10894 (Cre1 positive); lane 2, 'Prins'/AUS10894 (NIL) (Cre1 positive); lane 3, 'Prins' (Cre1 negative); and lane 4, 'Prins'/Loros (NIL) (Cre1 positive). Each set of DNA samples was digested with $\mathrm{DraI}, \mathrm{NdeI}$, and XbaI restriction endonucleases as indicated. Fragments were probed A, with G035-5'and B, with a partial cDNA of G035 that contains all of exon 2 and G035-3'. Filled arrows indicate the polymorphic hybridizing bands associated with the resistant allele. Open arrows indicate polymorphic bands associated with the susceptible allele. 
enzyme and probe combinations analyzed, the diagnostic Cre 1associated RFLP that were defined in the NIL were also present in all varieties of wheat carrying Crel resistance (Fig. 3).

The inheritance of the Crel-associated RFLP was investigated using the Frame $\times$ Silverstar doubled-haploid family, of which parental line Silverstar carries Crel resistance. In 96 doubled-haploid lines analyzed, the diagnostic RFLP generated with the different probe and enzyme combinations were inherited as a set and cosegregated exactly with nematode resistance. On the basis of either the G4 or G035 regions used as a probe to detect the diagnostic RFLP (Table 1) that were inherited as a unit in a segregating family, we designated the locus as Xcsl107. Thus, the set of diagnostic NBS-LRR RFLP characteristic of $\mathrm{Cre} 1$ nematode-resistant wheats constitute a haplotype at the Xcsl107 locus. Over 4,000 wheat lines from diverse backcross families segregating for $\mathrm{Crel}$ were also tested for segregation of the Xcsl107 diagnostic haplotype. The presence or absence of the diagnostic haplotype at the Xcsl107 locus correlated precisely with the resistance or susceptible phenotypes, respectively.

The diagnostic haplotype was used as a genotypic reference to evaluate wheat varieties for the presence of Crel nematode resistance. In a survey of nearly 2,000 wheat lines from Mediterranean, North African, and Middle Eastern regions for CCN resistance, McKay (1994) identified six wheat landraces that were resistant to the Australian CCN pathotype. However, it was unknown which one or more of the $\mathrm{CCN}$ resistance genes they carried. Using the diagnostic probe and enzyme combinations, four of the six landraces analyzed carried the Xcsl107 diagnostic haplotype for Crel (AUS4918 and AUS7639 from Iraq, AUS7869 and AUS13807 from Afghanistan), the line AUS4930 from Iraq carried only one of the gene members (RFLP derived from S33 clone; Table 1) associated with the diagnostic haplotype for Cre 1, and one of the landraces (AUS 10938 from Iran) did not have any bands associated with the Crel haplotype (data not shown).

\section{Molecular characterization}

\section{of the Xcsl107 locus that cosegregates with $\mathrm{Cre1}$.}

To analyze the members that constitute the haplotype of the Xcsl107 locus in Crel-containing wheats, a genomic lambda DNA library was made from a Crel-resistant wheat line and hybridized with probes G035-5' and G035-3' corresponding to the regions of exons 1 and 2 (Fig. 1). Lambda

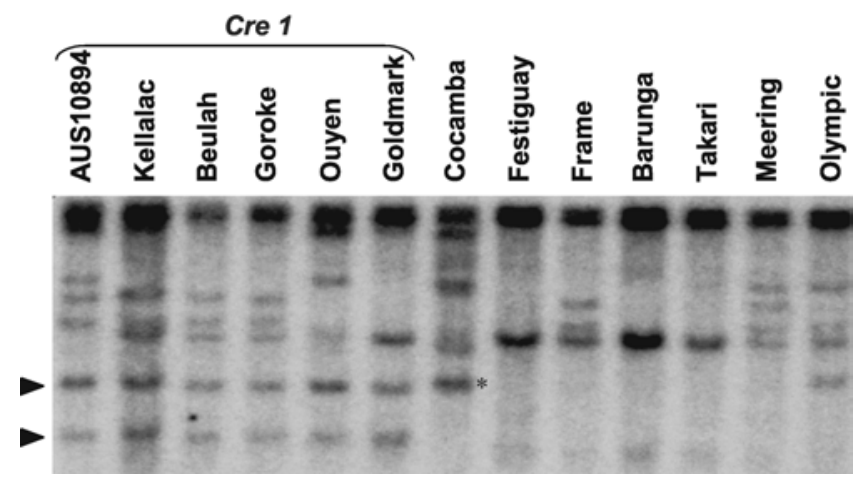

Fig. 3. Genomic DNA hybridization of Crel-resistant and non-Crel varieties of hexaploid wheat. NdeI restricted genomic DNA blot probed with a partial cDNA of G035 that contains all of exon 2 and G035-3'. The first six lanes contain wheat with $\mathrm{Crel}$ nematode resistance, and the next seven contain non-Crel-carrying wheat. The diagnostic bands that associate with the Crel phenotype are indicated with arrows. The DNA fragment marked (*) from the wheat variety Cocamba appears to be a similar size to the diagnostic fragment associated with Crel but is marginally larger in size and not linked to Crel. clones that hybridized with both probes and, thus, likely to carry full-length genes were recovered and compared for the presence of RFLP defining the diagnostic haplotype in Cre 1resistant genomic DNA in order to match lambda clones with polymorphic bands. Three lambda clones, called S33, M59, and M19, associated with some of the bands defining the diagnostic haplotype present in Crel wheats (Table 1) were subcloned and sequenced. Attempts were also made to characterize parts of subclones that detect a specific gene member of the constituent haplotype from the Xcsl107 locus when used in hybridization experiments with total wheat genomic DNA, to provide a basis for the potential development of "STS"-based diagnostic assay. An 800-bp fragment, S33-0.8, located $2.4 \mathrm{~kb}$ from the predicted 3' end of the S33 gene was used in further genomic DNA hybridization analysis, resulting in a simplification of the banding pattern when compared with that with the probes from the predicted coding regions (Fig. 4). The pattern was reduced from 9 to 12 bands to two bands (Fig. 4), with the probe derived from the S33-0.8 fragment. One of these bands is linked to Crel and provides a simple marker for the resistance gene. The second band is derived from the susceptible parent 'Prins'. This band is most likely from the D genome homologue based on the following evidence. First, only the Crel diagnostic band is detected in durum wheat (AB genomes) carrying Cre 1. Second, the probe hybridizes to a bacterial artificial chromosome (BAC) clone derived from the D genome of Aegilops tauschii containing the G12 NBS-LRR gene (data not shown). The landrace AUS 4930, which was shown to only have one band associated with the Xcsl107 haplotype diagnostic for Crel, was also shown to carry the S33 gene member by hybridization to S33-0.8.

The gene structure for each of the clones S33, M59, and M19 isolated from the Xcsl107 locus was predicted, based on homology alignment to the G035 NBS-LRR sequence from which a full-length cDNA and corresponding genomic clone was characterized (GenBank accession numbers AY124651 and AAC05834). The gene structure consisted of two exons and two introns (Fig. 1). The size of the intron separating exons 1 and 2 predicted for each gene member is $0.75 \mathrm{~kb}$ for G035, $3 \mathrm{~kb}$ for S33, $6.6 \mathrm{~kb}$ for M59, and $9 \mathrm{~kb}$ for M19, as determined by reverse transcription-polymerase chain reaction. The S33 lambda subclone is around $13 \mathrm{~kb}$, M19 is $20 \mathrm{~kb}$, and M59 is $18 \mathrm{~kb}$.

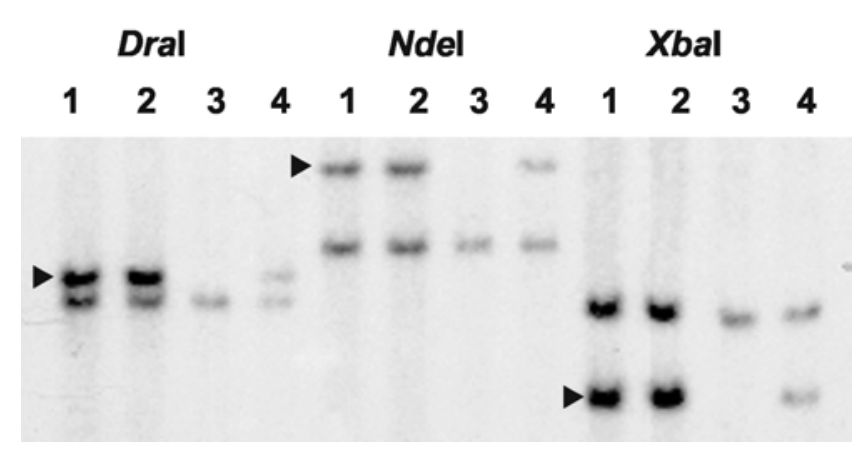

Fig. 4. Restriction fragment length polymorphism analysis of the wheat near-isogenic lines and parental source of $\mathrm{Crel}$ probed with fragment S33-0.8. Lane 1, AUS10894 (Cre1 positive); lane 2, 'Prins'/10894 (NIL) (Crel positive); lane 3, 'Prins' (Crel negative); and lane 4, 'Prins'/Loros (NIL) (Crel positive). Each set of DNA samples was digested with $\mathrm{DraI}, \mathrm{NdeI}$, and $\mathrm{XbaI}$ restriction endonucleases, as indicated. DNA blot is under stringent posthybridization washes $(0.1 \times \mathrm{SSC}(1 \times \mathrm{SSC}$ is $0.15 \mathrm{M} \mathrm{NaCl}$ plus $0.015 \mathrm{M}$ sodium citrate), $1 \%$ sodium dodecyl sulfate for $20 \mathrm{~min}$ ). Filled arrows indicate the S33 gene member at the Xcsl107 locus. 
Homeology between $\mathrm{Cre} 1$ and $\mathrm{Cre} 3$ loci.

Of the 13 DNA markers from homeologous group 2L tested for polymorphism between the parental lines Silverstar (Cre I) and Frame of the doubled-haploid family, three markers, consisting of BCD1231, glk605, and CDO36, were polymorphic for chromosome $2 \mathrm{BL}$ and were used to ascertain the marker order relative to Crel. BCD1231 is a partial cDNA clone originally derived from barley and is the most distally mapped marker on wheat group 2L chromosomes (Nelson et al. 1995; McGuire and Qualset 1997). The hybridization pattern of BCD1231 in wheat was complex (data not shown). We isolated the full-length homologue of BCD1231 from a wheat D genome cDNA library (GenBank accession number AF522460; 85\% sequence identity) in an attempt to use different parts of the sequence to generate probes that were less complex. The D genome equivalent of BCD1231 was designated csAtPPR5, as the deduced amino acid sequence showed similarity to a region contained in a precursor of pathogenesisrelated protein 5 (PR5) from rice. The clone csAtPPR5, when used as a probe, was shown to be part of the complex BCD1231 gene family on group 2L chromosomes and was tightly linked to the Crel and Cre3 loci (Fig. 5).

Segregation analysis of the doubled-haploid family Frame $x$ Silverstar, using the G035-5' probe-DraI restriction endonuclease combination, to detect the Xcsl107 diagnostic haplotype for $\mathrm{Cre} 1$ and the group 2L DNA markers revealed the following recombination frequencies: Xcsl107 haplotype/BCD1231 (csAtPPR5), 0.0\%; Xcsl107 haplotype/CDO36, 7.4\%; and Xcsl107 haplotype/glk605, 43.6\%. The resultant gene order for these loci on chromosome 2BL was Xcsl107/BCD1231 (csAtPPR5) to $7.5 \mathrm{cM}-\mathrm{CDO} 36$ to $56.1 \mathrm{cM}-\mathrm{glk} 605$ (Fig. 5) and
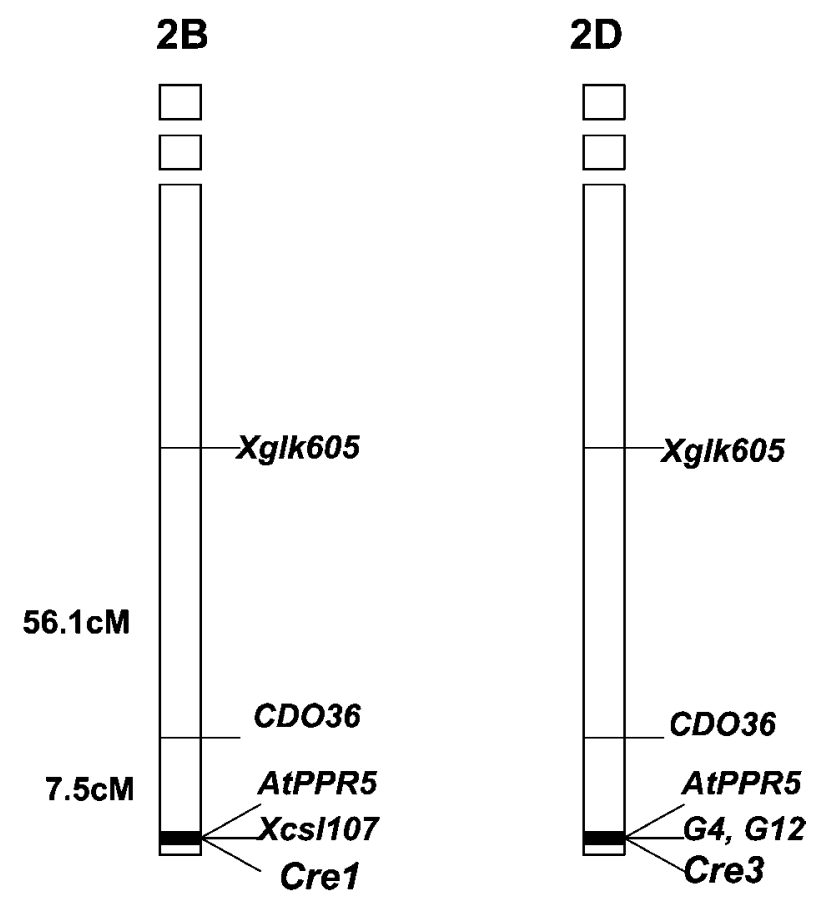

Fig. 5. Partial genetic linkage map of chromosomes $2 \mathrm{BL}$ and $2 \mathrm{DL}$, showing DNA marker order relative to the Crel and Cre3 loci. The 2B map is derived from the doubled-haploid family Frame $\times$ Silverstar and segregates for the diagnostic haplotype at the Xcsl107 locus. The map distances (centiMorgans) were determined using Mapmaker version 2 (Lander et al. 1987). In the chromosome 2D map the tight linkage between csAtPPR5 and G4/G12 sequences that cosegregate with $\mathrm{Cre} 3$ was determined from the cross between Aegilops tauschii accessions AUS18913 $($ Cre 1) $\times$ CPI110856. The relative order of markers with respect to CDO36 and Xglk605 was deduced from the consensus map of chromosome 2D (McGuire and Qualset 1997). was syntenic to the previously determined order for chromosome 2DL. Tight linkage was also observed between G4 and BCD1231/csAtPPR5 in an F2 family of 150 individuals segregating for $\mathrm{Cre} 3$ nematode resistance, in which no recombinants were recovered between BCD1231/csAtPPR5 and G4, which cosegregates with the Cre3 locus on 2DL. However, physical mapping in a BAC contig of approximately $200 \mathrm{~kb}$ previously assembled containing G4 and G12 sequences (Moullet et al. 1999) showed that it did not contain the BCD1231-related csAtPPR sequences.

\section{DISCUSSION}

Gene sequences cosegregating with the $\mathrm{Cre} 3$ locus, such as G4 and G12, or closely related gene members, such as G035, that are used in DNA hybridization experiments can detect homologues at syntenic and nonsyntenic locations in the wheat genome. These nonsyntenic regions are located on the short arm of chromosome $2 \mathrm{~A}$ and the long arms of chromosomes $5 \mathrm{~A}$ and 5D (Ogbonnaya et al. 2001a; Seah et al. 2000), which incidentally carried CCN resistance genes, Cre 5 and Cre6, respectively. However, these $\mathrm{CCN}$ genes were from introgressed alien segments derived from Aegilops ventricosa, which do not readily recombine with the wheat chromosomes, and so it is not possible to accurately link the homologs to Cre 5 and Cre6 based on normal meiotic recombination events. Homologues of the G4 and G12 NBS-LRR sequences identified in the present study from a syntenic region on chromosome $2 \mathrm{BL}$ located at the Xcsl107 locus were shown to define a haplotype associated with the presence of Crel resistance in wheat. Close identity between G12 and S33 sequences cosegregating with the Cre3 and $\mathrm{Cre} 1$ loci, respectively, was deduced in their $3^{\prime}$ flanking region under highly stringent hybridization conditions using an S33-specific probe. At least four gene members of the Xcsl107 diagnostic haplotype for Crel were identified in DNA hybridization experiments, of which three gene members were isolated and characterized in this study. Clustering of disease resistance gene sequences at a locus, similar to the observation found at the Xcsl107 locus is common among plants (Hulbert et al. 2001).

The Crel locus was previously placed within a proximal region relative to the centromere on wheat chromosome $2 \mathrm{BL}$ delineated at the distal end by the marker, glk605 (Williams et al. 1994). Our results using the markers glk605, CDO36, and BCD1231or its homologue csAtPPR5 reveal that Crel is more distal than either glk605 or CDO36 and is tightly linked to csAtPPR5/BCD1231, which is the most distal marker for chromosome 2BL (Fig. 5). The Cre3 locus has been established to be distally located on chromosome 2DL (Eastwood et al. 1994; Lagudah et al. 1997), and, using flanking markers as well as distantly placed markers, we confirmed the colinearity of the nematode resistance genes and markers on chromosomes $2 \mathrm{BL}$ and 2DL. These results establish the tight linkage between csAtPPR5/BCD1231 with the Cre 1 and $\mathrm{Cre} 3$ loci, suggesting a homeolocus relationship. In addition, overlapping resistance specificity between $\mathrm{Crel}$ and $\mathrm{Cre} 3$ against $\mathrm{H}$. avenae pathotypes have been reported (Eastwood 1995; Rivoal et al. 2001).

The NBS-LRR gene members that define the haplotype at the Xcsl107 locus that cosegregates with Crel provide a tool that allows the screening of wheat varieties for Crel nematode resistance at a molecular level. Routine marker-assisted selection for Australian and some European wheat lines segregating for Crel nematode resistance are being carried out using this method (Ogbonnaya et al. 2001b). The diagnostic haplotype was also used to help determine the type of resistance that a number of landrace wheats carried. Most were found to carry Crel resistance. In one landrace, AUS4930, only one of the 
gene members identical in size to S33 was observed from the diagnostic haplotype associated with Cre1. This association with one gene member may indicate a divergence or deletion of other gene members to constitute a variant haplotype present in AUS4930 at the Xcsl107 locus. Further genetic analysis of a segregating family involving AUS4930 is required to ascertain whether other gene members cosegregate with the sole S33 NBS-LRR member.

\section{MATERIALS AND METHODS}

\section{Plant material and nematode bioassays.}

Wheat genetic stocks of near-isogenic lines of $\mathrm{Cre} 1$ derived from either cv. Loros or Aus 10894 in cv. Prins background (developed and supplied by J. MacKey, Uppsala, Sweden), 96 doubled-haploid lines segregating for the presence or absence of $\mathrm{Cre} 1$ from the cross Frame $\times$ Silverstar, and six bread wheat landraces from Iraq and Afghanistan (AUS4918, AUS7639, AUS7869, AUS13807, AUS4930) with resistance to CCN but of unknown Cre gene status were analyzed in the study. A wide range of wheat cultivars and lines with and without Crel nematode resistance from Australia and Europe were used to test the cloned genes as probes to establish the DNA fingerprint or haplotype of Crel-containing wheats. The Cre 1 nematode-resistant wheat cultivars used were: Kellalac, Goldmark, Ouyen, Goroke, Beulah, Silverstar, RE8607, Loros, AUS 10894,VI 252, and VI 727. Non-Crel-containing wheat cultivars used were: Almatense, Aroona, Arrino, Avocet, Banks, Barunga, Bass, Bindawarra, Bodallin, Cadoux, CD87, Chinese Spring, Condor, Corrigin, Cranbrook, Crest, Cunningham, Currawong, Dagger, Diamondbird, Egret, Eradu, Excalibur, Falcon, Festiguay, Flinders, Frame, Gabo, Gamenya, Genaro, Gordon, Gutha, Halberd, Hardi, Hartog, Heron, Janz, Jing Hong, Katepwa, Kewell, Kiata, Kite, Klasic, Krichauff, Kulin, Lawson, Machete, Madden, Matong, ME71, Meering, Mendos, Millewa, Minto, Moisson, Molineux, Neepawa, Olympic, Osprey, Oxley, Patterson, Pinnacle, Purple Straw, QT2200-20, Quarrion, RAC820, Reeves, Schomburgk, Songlen, Spear, Stilletto, Sunco, Suneca, Sunkota, Sunlin, Sunstar, Takari, Tammin, Tasman, Tincurrin, Vulcan, Warbler, Warigal, Wyuna, and ZL59B. Backcross-derived plants $(4,000)$ segregating for Cre 1 nematode resistance from 40 different crosses were also analyzed for the presence or absence of the diagnostic haplotype for Cre1. A minimum of 100 lines selected from each of the 40 different $\mathrm{BC} 1 \mathrm{~F} 4$ families were phenotyped for $\mathrm{CCN}$ resistance and were genotyped using markers that cosegregate with $\mathrm{Cre} 1$ resistance as part of the marker-assisted breeding program at the Department of Primary Industries, Horsham, Victoria, Australia.

Pot bioassays for classifying plants as resistant or susceptible to $H$. avenae pathotype Ha13 (Australian strain) were as described by Eastwood and associates (1994). An F2 family from a cross between Aegilops tauschii accessions AUS18913 $(\mathrm{Cre} 3) \times \mathrm{CPI} 110856$ segregating for $\mathrm{Cre} 3$ resistance was used to determine other markers linked to the $\mathrm{Cre} 3$ locus.

\section{DNA and RFLP analysis.}

DNA extractions and RFLP analyses were as previously described (Lagudah et al. 1991a and b). Genomic DNA extracted from the near-isogenic lines, Frame $\times$ Silverstar population, wheat landraces, cultivars, and breeding lines were digested with a selection of DraI, NdeI, and XbaI for RFLP analysis. Probes used in the RFLP analysis were NBSLRR gene members G4 and G12, which cosegregate with the Cre3 locus, and a closely related gene member, G035, was also identified in the course of screening the Aegilops tauschii (accession number AUS1893) genomic lambda and
BAC libraries as previously described (Lagudah et al. 1997; Moullet et al. 1999). Pulse field gel electrophoretic analysis of BAC clones from Aegilops tauschii were as described by Moullet and associates (1999). A cDNA library from Aegilops tauschii was also screened in an attempt to recover full-length cDNA clones, using SuperScript II RNase $\mathrm{H}^{-}$ (Life Technologies, Gaithersburg, MD, U.S.A.) as the reverse transcriptase enzyme. A genomic DNA library from a tetraploid wheat derivative carrying $\mathrm{Crel}$ resistance selected from a backcross population derived from an initial pentaploid progeny of Cham (4x)/Re8607 (6x Cre1 source) was constructed in lambda GEM11 from size-fractionated partial digests of genomic DNA cleaved with Sau3A and MboI according to the manufacturers instructions (Promega, Madison, WI, U.S.A.). The G4-related gene member (G035) was used to make probes to analyze the library and the wheat genetic stocks. The specific gene regions of G035 and a partial cDNA that were used as probes are shown in Figure 1. Thirteen markers that were previously mapped to the long arm of chromosome 2B (glk 605, BCD1779, BCD1231, CDO36, BCD1095, PSR390, PSR540, PSR609, PSR934, CDO1328, CDO1417, $\mathrm{BCD} 445$, and $\mathrm{BCD} 135)$ in addition to the G4-related probes were used to determine the precise location of the diagnostic NBS-LRR RFLP haplotype for Cre1, using the Frame $\times$ Silverstar doubled-haploid family. The locus on chromosome $2 \mathrm{BL}$ containing the diagnostic NBS-LRR gene members cosegregating with $\mathrm{Crel}$ was designated XcsllO7 in accordance with the rules of the wheat gene catalogue (McIntosh et al. 1998) for DNA markers $(X)$, laboratory designation $(c s l)$, and probe number (107), as defined by RFLP detected using either DraI, NdeI, or XbaI (Table1) with the probes G4-5', G035-5', and G035-3'. Sequencing and analysis of the isolated clones were as described by Seah and associates (2000).

\section{Primers and PCR amplification conditions.}

Primer sets used to amplify fragments from the G035 clone to make probes were G035 5' F, 5'TGCAGCCTAGCCTTCTC CCCA3', and G035 5' R, 5'GTAGCTTCATATATATGGAA C3', for exon 1 and CreconF1, 5'ATCTGATCAACTTGCGGC AT3', and Crecon R1, 5'ACTCTGACTCCGATTCCAAG3', for exon 2. Amplification conditions were as described by Seah and associates (1998).

\section{ACKNOWLEDGMENTS}

We are grateful to S. Chandramohan, L. Viccars, and J. Wilson for excellent technical support, to Graingene (a research alliance between CSIRO, AWB Limited, GRDC, and Syngenta Seeds) for financing the project, to J. Jahier for supplying seeds of RE8607 and the selfed progeny of the pentaploid cross, and to J. Mackey for the near-isogenic lines in 'Prins'.

\section{LITERATURE CITED}

Cai, D., Kleine, M., Kifle, S., Harloff, H. J., Sandal, N. N., Marcker, K. A., Klein-Lankhorst, R. M., Salentijn, E. M. J., Lange, W., Stiekema, W. J., Wyss, U., Grundler, F. M. W., and Jung, C. 1997. Positional cloning of a gene for nematode resistance in sugar beet. Science 282:2012-2018.

Eastwood, R. F. 1995. Genetics of resistance to Heterodera avenae in Triticum tauschii and its transfer to bread wheat. Ph.D. thesis, University of Melbourne, Australia.

Eastwood, R. F., Lagudah E. S., and Appels, R. 1994. A directed search for DNA sequences tightly linked to cereal cyst nematode resistance genes in Triticum tauschii. Genome 37:311-319.

Ernst, K., Kumar, A., Kriseleit, D., Kloos, D.-U., Phillips, M. S., and Ganal, M. W. 2002. The broad-spectrum potato cyst nematode resistance gene (Hero) from tomato is the only member of a large gene family of NBS-LRR genes with an unusual amino acid repeat in the LRR region. Plant J. 31:127-136. 
Hulbert, S. H., Webb, C. A., Smith, S. M., and Sun, Q. 2001. Resistance gene complexes: Evolution and utilization. Annu. Rev. Phytopathol. 39:285-312.

Lagudah, E. S., Appels, R., Brown, A. H. D., and McNeil, D. 1991a. The molecular-genetic analysis of Triticum tauschii-the D genome donor to hexaploid wheat. Genome 34:375-386.

Lagudah, E. S., Appels, R., Brown, A. H. D., and McNeil, D. 1991b. The Nor-D3 locus of Triticum tauschii: Natural variation and linkage to markers in chromosome 5. Genome 34:387-395.

Lagudah, E. S., Moullet, O., and Appels, R. 1997. Map based cloning of a gene sequence encoding a nucleotide binding domain and leucine rich region at the $\mathrm{Cre} 3$ nematode resistance locus of wheat. Genome 40:659-665.

Lagudah, E. S., Moullet, O., Ogbonnaya, F., Seah, S., Eastwood, R. F., Appels, R., Jahier, J., Lopez-Brana, I., and Delibes, A. 1998. Cloning of disease resistance gene sequences at loci conferring cyst nematode resistance in wheat. Pages 184-186 in: Proceedings of the 9th International Wheat Genetics Symposium, Vol. 1. A. E. Slinkard, ed. University Extension Press, Saskatoon, Sakatchewan, Canada.

Lander, E. S., Green, P., Abrahamson, J., Barlow, A., Daly, M. J., Lincoln, S. E., and Newburg, L. 1987. MAPMAKER: An interactive computer package for constructing primary genetic linkage maps of experimental and natural populations. Genomics 1:174-181.

McIntosh, R. A., Hart, G. E., Devos, K. M., Gale, M. D., and Rogers, W. J. 1998. Catalogue of gene symbols for wheat. Pages 1-236 in: Proceedings of the 9th International Wheat Genetics Symposium, Vol. 5. A. E. Slinkard, ed. University Extension Press, Saskatoon, Sakatchewan, Canada.

McGuire, P. E. and Qualset, C. O. 1997. Progress in genome mapping of wheat and related species. Genetic Resources Conservation Program, Division of Agriculture and Natural Resources, University of California, Davis, U.S.A.

McKay, A. 1994. Pages 1-15 in: Genetics of Resistance and Tolerance to $\mathrm{CCN}$ in Wheat. Final report for the Grains Research and Development Corporation of Australia project number DAS 6C. Grains Research and Development Corporation, Canberra, Australia.

Milligan, S. B., Bodeau, J., Yaghoobi, J., Kaloshian, I., Zabel, P., and Williamson, V. M. 1998. The root-knot nematode resistance gene Mi from tomato is a member of leucine zipper, nucleotide binding, leucine rich repeat family of genes. Plant Cell 10:1307-1319.

Moullet, O., Zhang, H. B., and Lagudah, E. S. 1999. Construction and characterisation of a large DNA insert library from the D genome of wheat. Theor. Appl. Genet. 99:305-313.

Nelson, J. C., Van Deynze, A. E., Autrique, E., Sorrells, M. E., Lu, Y. H., Merlino, M., Atkinson, M., and Leroy, P. 1995. Molecular mapping of wheat. Homoeologous group 2. Genome 38:516-524.

Nielsen, C. H. 1966. Studies on the inheritance of resistance to cereal cyst nematode (Heterodera avenae) in wheat. Nematologica 12:575-578.
Nilsson-Ehle, N. H. 1920. Ueber Resistenz gegen Heterodera schactii be gewissen Gerstensorten, ihre Verebungsweise und Bedeutungfur die Praxis. Hereditas 1:1-34.

O’Brien, P. C., Fisher, J. M., and Rathjen A. J. 1980. Inheritance of resistance in two wheat cultivars to an Australian population of Heterodera avenae. Nematologica 26:69-74

Ogbonnaya, F. C., Seah, S., Delibes, A., Jahier, J., Lopez-Brana, I., Eastwood, R. F., and Lagudah, E. S. 2001a. Molecular-genetic characterisation of a new nematode resistance gene in wheat. Theor. Appl. Genet. 102:623-629.

Ogbonnaya, F. C., Subrahmanyam, N. C., Moullet, O., de Majnik, J., Eagles, H. A., Brown J. S., Eastwood, R. F., Kollmorgen, J., Appels, R., and Lagudah, E. S. 2001b. Diagnostic DNA markers for cereal cyst nematode resistance in bread wheat. Aust. J. Agric. Res. 52:13671374

Rivoal, R., Bekal, S., Valette, S., Gauthier, J. P., Hadj Fradj, M. M. Mokabli, A., Jahier, J., Nicol, J., and Yahyaoui, A. 2001. Variation in reproductive capacity and virulence on different genotypes and resistance genes of Triticeae, in the cereal cyst nematode species complex. Nematology 3:581-592.

Seah, S., Sivasithamparam, K., and Lagudah, E. S. 1998. Cloning and characterisation of a family of disease resistance gene analogs from wheat and barley. Theor. Appl. Genet. 97:937-945.

Seah, S., Spielmeyer, W., Jahier, J., Sivasithamparam, K., and Lagudah, E. S. 2000. Resistance gene analogs within an introgressed chromosomal segment derived from Triticum ventricosa that confers resistance to nematode and rust pathogens in wheat. Mol. Plant-Microbe Interact. 13:334-341.

Slootmaker, L. A. J., Lange, W., Jochemsen, G., and Schepers, J. 1974. Monosomic analysis in bread wheat of resistance to cereal root eelworm. Euphytica 23:497-503.

Spielmeyer, W., Robertson, M., Collins, N., Leister, D., Schulze-Lefert, P., Seah, S., Moullet, O., and Lagudah, E. S. 1998. A superfamily of disease resistance gene analogs is located on all homoeologous chromosome groups of wheat (Triticum aestivum). Genome 41:782-788.

Williams, K. J., Fisher, J. M., and Langridge, P. 1994. Identification of RFLP markers linked to the cereal cyst nematode resistance gene (Cre) in wheat. Theor. Appl. Genet. 89:927-930.

Van der Vossen, E. A., Van der Voort, J. N., Kanyuka, K., Bendahmane, A., Sandbrink, H., Baulcombe, D. C., Bakker, J., Stiekema, W. J., and Klein-Lankhorst, R. M. 2000. Homologues of a single resistance-gene cluster in potato confer resistance to distinct pathogens: A virus and a nematode. Plant J. 5:567-576.

\section{AUTHOR-RECOMMENDED INTERNET RESOURCE}

GrainGenes wheat database: wheat.pw.usda.gov/ggpages/maps.shtml\#wheat 